\title{
Study on Fiber Optical Vibration Monitoring System for Electromechanical Equipment
}

\author{
Shujuan $\mathrm{Li}^{1,2, \text { a }}$, Xiaohui Liu ${ }^{1,2, \mathrm{~b}}$, Li Min ${ }^{1,2, \mathrm{c}}$ and Chang Wang ${ }^{1,2, \mathrm{~d}^{*}}$ \\ ${ }^{1}$ Shandong Key Laboratory of Optical Fiber Sensing Technology, Jinan \\ ${ }^{2}$ Laser Research Institute of Shandong Academy of Sciences, Jinan \\ asdlshj@126.com, ’windnuaa@126.com, cip_99@163.com, ${ }^{\mathrm{d}}$ wang960100@163.com
}

\begin{abstract}
Keywords: Mechanical \& Electrical Equipment; Vibration; Fiber Bragg Grating.
\end{abstract}
\begin{abstract}
To improve equipment utilization and guarantee long-term reliable efficiently running for mechanical and electrical equipment, fiber-optic sensing technology is applied to construct mechanical and electrical equipment fiber-optic vibration monitoring system, which can achieve real-time online monitoring and early warning of device status, and provide reliable data for the device fault diagnosis. Field test and comparison showed that the vibration monitoring system meets the equipment needs for vibration monitoring.
\end{abstract}

\section{Introduction}

Safety monitoring of mechanical \& electrical equipment play an extremely important role in safety production to support device running normally and prevent operation accidents. Operating experience shows that mechanical \& electrical equipment failure caused by super-vibration, overheating, overpressure and corrosion is a major factor causing a major accident. Mechanical vibration analysis is one of the most important methods to measure the operation of equipment. Machinery vibration condition monitoring can grasp the mechanical integrity degree and mechanical performance characteristics.

Currently, the state checks of mechanical \& electrical equipment mainly depend on the maintenance and test before running. While the devices are running, the state checks mainly depend on system's operating parameters monitoring and manual inspection. It is clearly that the device operation status assessments are lack of online real-time monitoring. Furthermore, manual inspection methods including infrared temperature measurement, electrical insulation measurement or motor bearing vibration could miss a lot of test points and has high error rate.. Major equipment usually has individual device to run statement online monitoring and use lots of electrical sensors. Thus it is difficult to deal with insulation, wiring complex and has poor ability to resist electromagnetic interference, which often leads to large measurement error, and is not conducive to fault monitoring and diagnosis.

The fiber optic sensor[1] as an optical measuring instrument has more advantages, such as high sensitivity and resolution, wide bandwidth, high response speed, large dynamic range, small size, long transfer distance, simply wiring, uncharged and anti-electromagnetic interference, anti-radiation, which make it suitable for flammable, explosive space and other harsh environments, etc.. And it is much easier to implement real-time fault monitoring and diagnosis.

This paper introduces the fiber-optic sensing technology used in electrical \& mechanical equipment vibration testing and realization of equipment real-time online status monitoring.

\section{Design of Vibration Sensing System for Electromechanical Equipment}

The system uses vibration sensors designed based on fiber Bragg grating (FBG). The Bragg wavelength of FBG changes with temperature and pressure. A wavelength demodulation system for recognition of the center wavelength under corresponding temperature, pressure, strain and other parameters is used to achieve a sensing function [2-5]. 
According to elastic fiber-optic effect and thermo-optic effect theory, the FBG center wavelength with strain and temperature changes in the relationship:

$$
\Delta \lambda_{B}=\lambda_{B}\left(1-P_{e}\right) \Delta \varepsilon+\lambda_{B}(\alpha+\xi) \Delta T
$$

Where, $\Delta \lambda_{B}$ is the change of center wavelength of reflected light caused by stress and temperature changing; $\Delta \varepsilon$ is the strain change; $\Delta T$ is the temperature change; $P_{e}$ is the elastic-optic coefficient of optical fiber; $\alpha$ is the fiber thermal expansion coefficient; $\xi$ is the thermo-optic coefficient of optical fiber.

By vibration transform devices, mechanical vibrations are changed into the optical fiber grating strain, consequently it is equivalent to testing dynamic or rapid response in vibration measurement. Vibration sensor structure model is shown in Fig. $1 . k_{1}$ is the elasticity of the fiber; $k_{2}$ is structure elasticity coefficient; $c$ is the medium damping coefficient; $m$ is quality of mass.

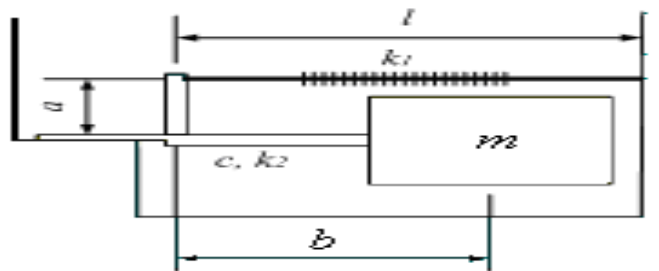

Figure 1. Mechanical model of the vibration sensor structure

The overall elasticity coefficient $k$ is:

$$
k=k_{2}+k_{1}\left(\frac{a}{b}\right)^{2}
$$

The dynamics equation of vibration sensing system subject to acceleration is:

$$
m \ddot{y}+c \dot{y}+k y=m a_{0} e^{i \omega t}
$$

Where: $\omega$ is the circular frequency acceleration, $a_{0}$ is the acceleration amplitude.

Let $\delta=\frac{c}{2 m}, \omega_{0}^{2}=\frac{k}{m}$, Then the above equation can be expressed as:

$$
\ddot{y}+2 \delta \ddot{y}+\omega_{0}^{2} y=\bar{F}
$$

$\delta$ is the relative damping coefficient, $\omega_{0}$ is the system natural frequency. The eigen solution of equation set:

$\bar{Y}=B e^{i(\omega t+\varphi)}$

Where $B$ is the mass amplitude, $\varphi$ the initial phase.

That

$$
\bar{Y}=\frac{\bar{F}}{-\omega^{2}+2 i \omega \delta+\omega_{0}^{2}}
$$

Let

$$
\bar{H}=\frac{1}{\omega_{0}^{2}-\omega^{2}+2 i \omega \delta}
$$

$\bar{H}(\omega)$ is complex frequency response, its modulus $|\bar{H}|$ and angular $\sigma$ are: 


$$
\begin{aligned}
& \bar{H}=\frac{1}{\sqrt{\left(\omega_{0}^{2}-\omega^{2}\right)^{2}+4 \delta^{2} \omega^{2}}} \\
& \sigma=-\arctan \frac{2 \delta \omega}{\omega_{0}^{2}-\omega^{2}}
\end{aligned}
$$

So

$$
\bar{Y}=\bar{H} \bullet \bar{F}=|\bar{H}| e^{i \sigma} \bullet a_{0} e^{i \omega t}=a_{0}|\bar{H}| e^{i(\omega t+\sigma)}
$$

Then the amplitude $\mathrm{B}$ is:

$$
B=a_{0}|\bar{H}|
$$

Strain $\varepsilon$ generated on the FBG is:

$$
\varepsilon=\frac{a B}{b l}=\frac{a_{0} a}{b l \omega_{0}^{2}}
$$

Strain - acceleration sensitivity coefficient $\mathrm{K}$ is:

$$
K=\frac{a}{b l \omega_{0}^{2}}=\frac{a m}{b l k}=\frac{a m}{b l} \bullet \frac{1}{k_{2}+(a / b)^{2} k_{1}}
$$

By appropriate choice of parameters such as elasticity coefficient $k$ and mass of the total system, we can design sensor operating in different frequency.

The acquisition of vibration signal is based on unbalanced Michelson interferometer demodulation. The fiber-optical unbalanced Michelson interferometer is designed based on traditional Michelson interferometry, which is used for transferring the wavelength containing vibration signal into phase, which is measured to determine vibration information.

The characteristic information corresponding to equipment status can be extracted by process of vibration signal. The useful signals need to be collected by feature extraction from various signals and then analyzed and processed. Afterwards the discrimination model and criterion are set up on account of the characteristic information of different parts to estimate the equipment operating state and evaluation area. Finally the judgment of equipment status will help make work plan and maintenance suggestion, for example replacing the bearing, dynamically balancing the rotor or continuing monitoring.

\section{Experiment Results}

The fiber-optic sensor and system are tested by standard vibration generator. The FBG sensor is fixed on the generator which generates a standard vibration signal of $40 \mathrm{~Hz}, 1.5 \mathrm{~V}$. Figure 2 show us the demodulated vibration signal, whose time domain profile is shown in Fig. 2 (a) and spectrogram is shown in Fig. 2 (b). 


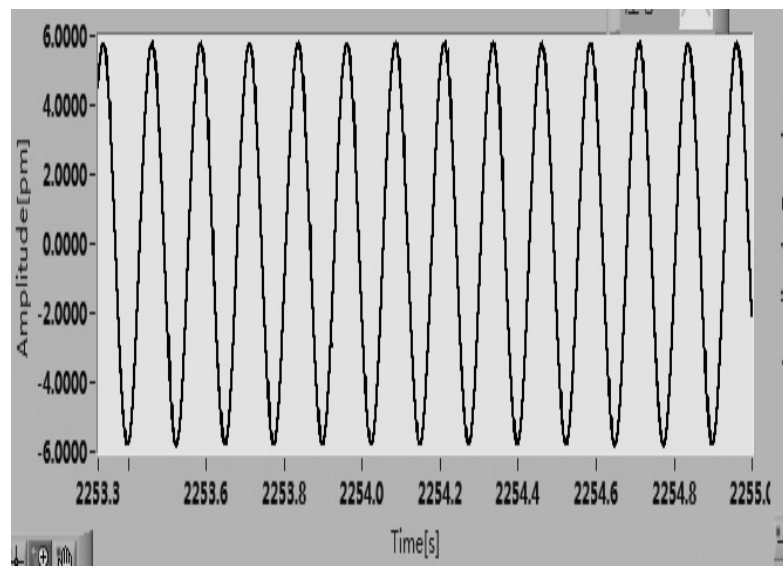

(a)

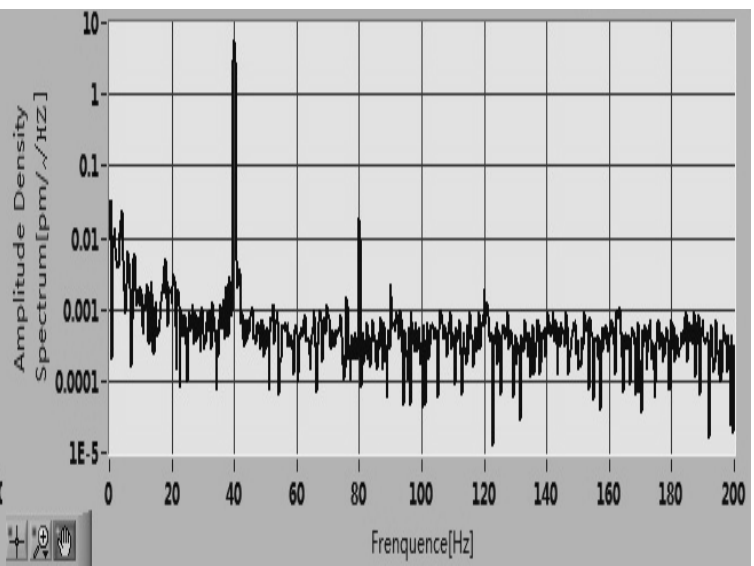

(b)

Figure 2. Vibration signal detected by fiber-optic sensing system

The FBG sensor is calibrated by use of standard B \& K4371 piezoelectric accelerometer then the sensitivity is imported into the system. Both the fiber-optic sensor and piezoelectric accelerometer are mounted on the vibration generator, which can generate standard vibration signal to measure the performance of fiber-optic sensor. When the $5 \mathrm{~Hz}$ signal is generating, the vibration signals measured by FBG senor and B\&K4731 accelerometer are shown in Fig. 3, which illustrated that the FBG sensor can aquire the exact vibration signal.

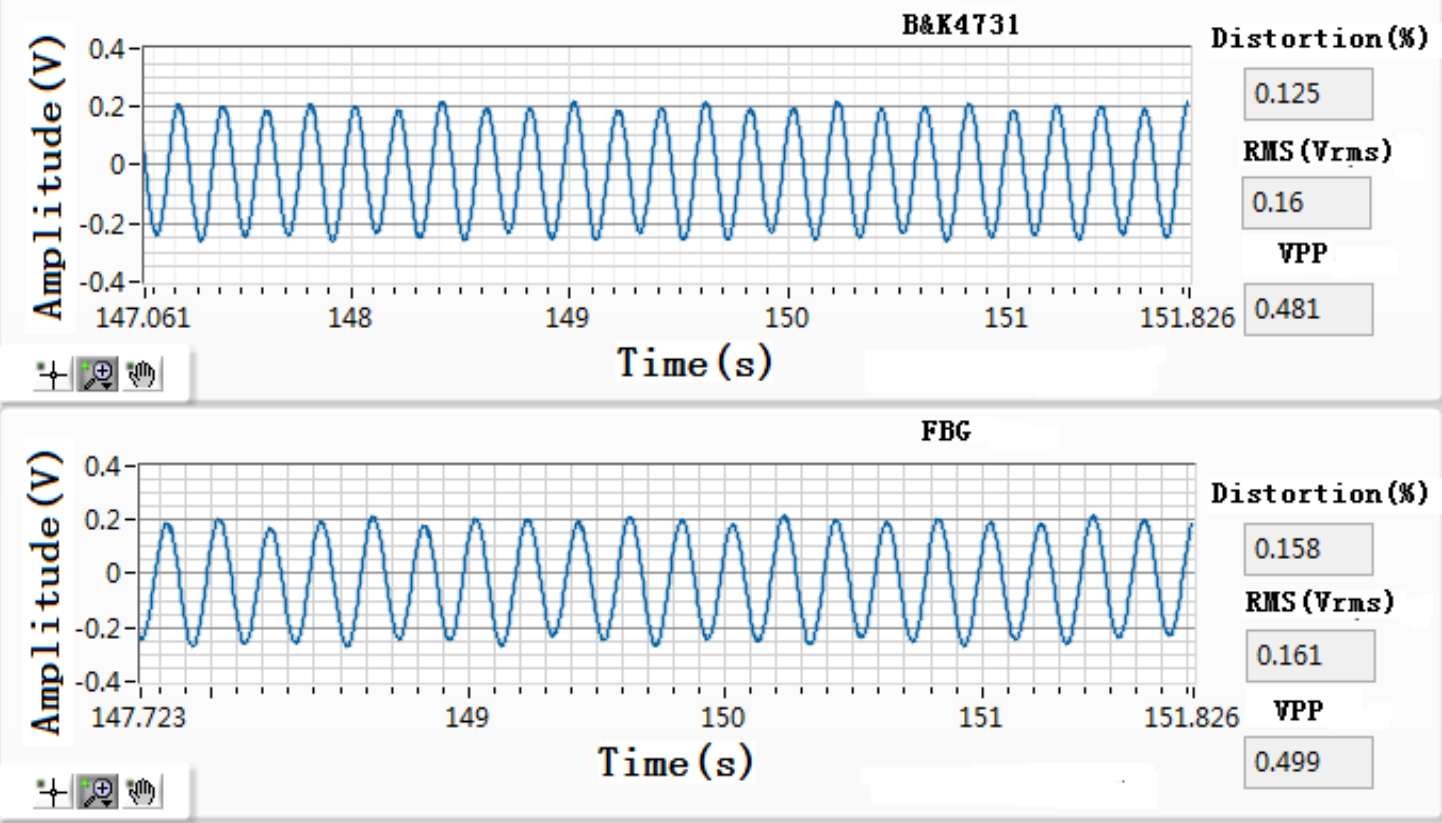

Figure 3. The vibration signal detected by FBG sensor and B\&K4731 accelerometer

The FBG sensors were mounted on the generator set for monitoring its operation status in the field test. The vibration signals detected by the fiber-optic sensing system were carried on frequency spectrum and phase analysis to estimate the operation status of the generator set, and then uploaded to the control room. Fig. 4 shows us an example of a set of vibration signal detected of the stator while the generator was running. 


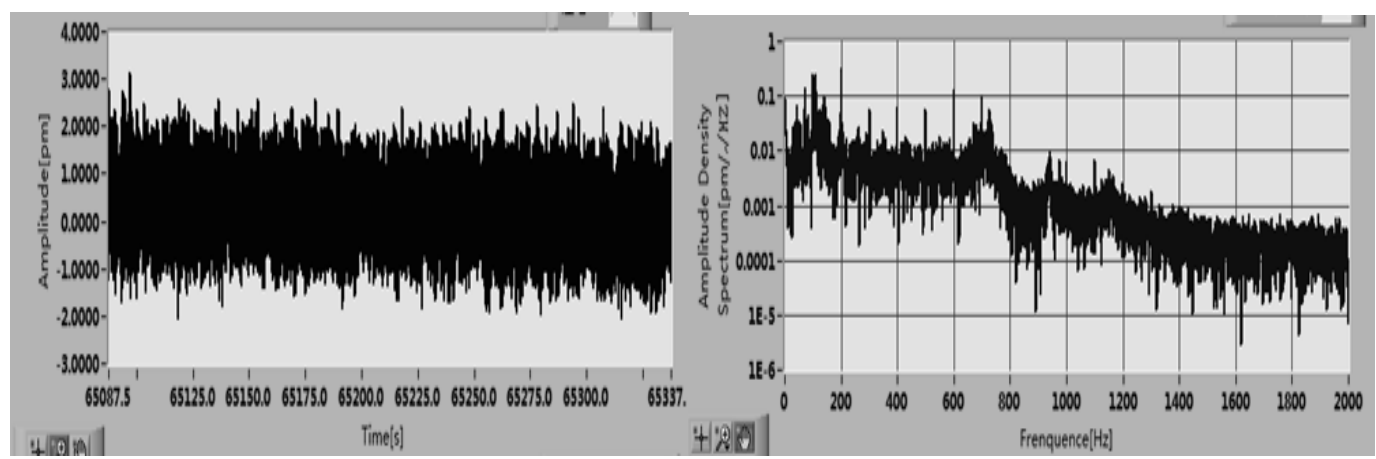

Figure 4. Vibration signal of the generator stator detected by FBG sensor

\section{Summary}

This paper studies the optical vibration detection in mechanical and electrical equipment condition monitoring applications, and the FBG sensor design. With a standard field test comparison, the experiment results show that this system can reflect real-time electrical and mechanical equipment operating status. The system gives the reliable data for the equipment fault diagnosis.

\section{Acknowledgements}

This work was supported by the National Natural Science Foundation of China (Nos. 61307101 and 61205083), the National Natural Science Foundation of Shandong province (No. ZR2013FL029), and key Research and Development Project of Shandong Province (Nos.2014GGX103005, 2015GSF115006, 2016GGx103041);

\section{References}

[1] K.O.Hill, Y.Fujii, D.C.Johnson,et al. Photosensitivity in optical fiber waveguide, Applied Physics Letters, 1978,32(10):647-649.

[2] Botsis J, Humbert L. Embedded fiber Bragg grating sensor for internal strain measurements in polymeric materials, Opt Lasers Eng, 2005, 42(3):491

[3] Cusano A, Cutolo A. Dynamic strain measurements by fiber Bragg grating sensor, Sens Actuators A, 2004, 110(1): 276

[4] Dongsheng Zhang, Dan Guo. Researches on Fiber Grating Vibration Sensor Based on Matching Filtering Demodulation, Chinese Journal of Sensors and Actuators.2007,20(2):311-313.

[5] Xianfeng Gong, Honggang Yi, Xiaomin Zhou. Low frequency fiber Bragg grating acceleration sensors, Journal of University of Science and Technology, Beijing, 2006, 28(1):75-77. 